\title{
Cellules souches
} pluripotentes de rat : so what?

John De Vos

Institut de Recherche en Biothérapie, Inserm U847, CHU de Montpellier, 80 , avenue A. Fliche, 34295 Montpellier, France. john.devos@inserm.fr
$>$ Les cellules souches embryonnaires (ES) font l'objet d'une large couverture médiatique en raison de leurs potentialités thérapeutiques mais aussi des questions éthiques qu'elles soulèvent chez l'homme. Mais bien avant que ces promesses et ces débats n'occupent le devant de la scène, ces cellules ont d'abord joué un rôle moins médiatisé et pourtant central en biologie moderne, comme outil pour la création des souris «knock-out» (KO) et transgéniques. C'est en effet par la manipulation génétique des cellules ES de souris et leur injection dans un embryon au stade de blastocyste, ou leur agrégation à un embryon au stade plus précoce de morula, que l'on obtient des souris chimères dont une partie des cellules, y compris germinales, est porteuse de la mutation génétique. Le croisement des souris chimères entre elles peut aboutir à des souris homozygotes pour la modification génétique, c'est-à-dire que chaque cellule porte alors l'altération génétique désirée. Celle-ci peut être soit l'invalidation d'un gène par recombinaison homologue (souris $\mathrm{KO}$ ) soit l'addition d'un gène supplémentaire (souris transgéniques). Cette technique a révolutionné la biologie en permettant de comprendre la fonction des gènes au cours du développement et ensuite dans le fonctionnement d'un mammifère adulte. Elle a également permis la création de nombreux modèles murins de pathologies humaines. Plus de 11000 gènes ont déjà été invalidés chez la souris, et des programmes internationaux sont en cours pour arriver à disposer des animaux $K O$ pour chacun des gènes de la souris [1, 2]. En 2007, le prix Nobel de médecine a été attribué à Martin Evans, Mario Capecchi et Oliver Smithies pour récompenser leurs travaux sur les cellules souches embryonnaires de souris et la recombinaison homologue, deux techniques dont le mariage avait abouti aux premières souris $\mathrm{KO}$ [3].

\section{Le truc pour réussir}

des cellules $\varepsilon S$ de rat

Mais jusqu'à présent, la technique ne s'appliquait qu'à la souris. En effet, l'impossibilité de dériver de véritables cellules ES chez le rat avait fait échouer les tentatives de création d'animaux KO chez cette espèce. Deux publications viennent de changer la donne et rapportent la dérivation de cellules souches embryonnaires de rat et montrent la possibilité d'obtenir des rats chimériques avec transmission germinale du patrimoine des ES. C'est l'équipe d'Austin Smith qui a apporté la solution du problème en établissant début 2008 de nouvelles conditions de culture des cellules ES murines, basées sur la combinaison de trois inhibiteurs chimiques $^{1}$ appelée $\ll 3 i »[4,5](\rightarrow)$. Alors

$(\rightarrow)$ Voir $\mathrm{m} / \mathrm{s} 2008$, $n^{\circ} 6-7$, p. 593 que les conditions de dérivation «classiques » des ES de souris utilisant un feeder de fibroblastes embryonnaires et le facteur de croissance LIF (leukemia inhibiting factor) n'avaient pas permis l'obtention d'ES de rat, les $3 i$ se sont avérés plus efficaces, entraînant l'expansion en culture de la masse cellulaire interne des

${ }^{1}$ Inhibiteurs de la voie GSK3/ $\beta$-caténine, de ERK phosphorylée et du récepteur de FGF4. blastocystes de rat et la dérivation de lignées de cellules souches embryonnaires de rat $[6,7]$. Cette application de la technologie des ES à une autre espèce de rongeur est une avancée considérable pour le développement de futures approches thérapeutiques humaines car elle ouvre la porte à l'obtention de rats KO et transgéniques. Or le rat est, pour de nombreuses maladies humaines, un bien meilleur modèle préclinique que la souris, en particulier pour la compréhension des pathologies comportementales et des pathologies multifactorielles.

\section{Animaux knock-out}

\section{via la technologie iPS}

Le développement d'une autre technologie, celle des cellules souches pluripotentes induites (iPS) [8] pourrait également aboutir à la création de rats KO. Deux équipes viennent en effet d'établir des lignées de cellules iPS de rat qui présentent (presque) toutes les caractéristiques des cellules souches embryonnaires: une signature d'expression génique de pluripotence, un caryotype normal, une prolifération prolongée - voire illimitée, mais un recul supplémentaire est requis - et une capacité de différenciation in vivo en cellules différenciées composant les tissus issus des trois feuillets embryonnaires [9-11]. Lorsqu'elles sont injectées dans des souris NOD/SCID (non-obese diabetic severe combined immunodeficient), ces iPS de rat forment des tératomes. Et l'injection d'iPS de rat dans des blastocystes de rat implantés dans l'utérus de femelles gravides a abouti à la naissance de rats dont le chimérisme est démontré par la couleur de la fourrure des animaux 


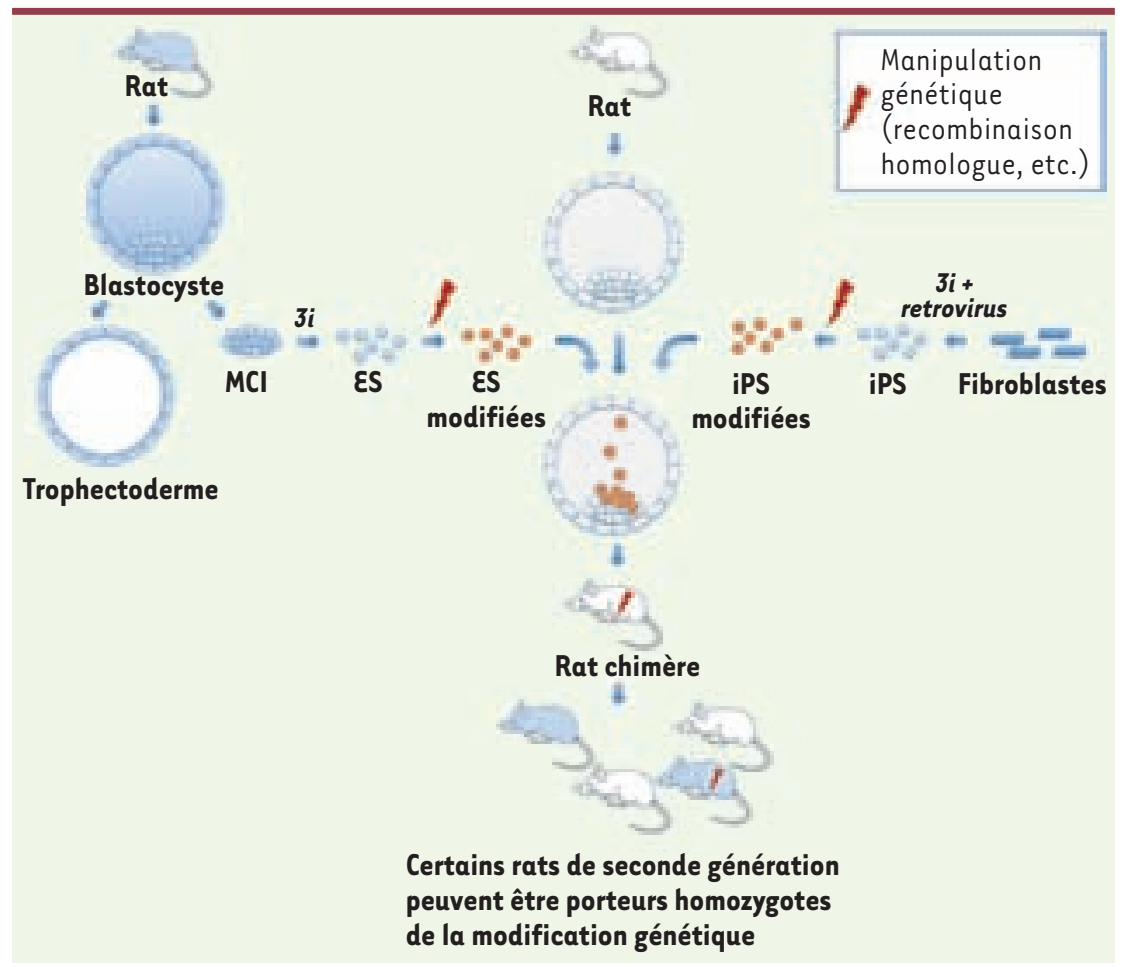

obtenus. Malheureusement, au cours de ces premières expériences, aucune transmission à la lignée germinale n'a été observée. Mais, compte tenu des résultats obtenus à partir des cellules $E S$ de rat d'une part, et de la possibilité de générer à partir d'iPS de souris des animaux chimères transmettant à leurs cellules germinales le patrimoine génétique des iPS d'autre part, il est vraisemblable qu'il soit possible à court terme de générer des rats $\mathrm{KO}$ ou transgéniques grâce à la technologie des iPS.

Une ménagerie d'animaux knock-out? Ces résultats permettent d'envisager la création d'animaux génétiquement modifiés par la technologie des iPS dès lors que la reprogrammation sera accessible pour une espèce animale donnée. La transgenèse chez les animaux n'était jusque-là possible que par manipulation génétique d'embryons par l'intermédiaire de vecteurs rétroviraux; c'est le cas du poisson Glofish, rendu fluorescent par l'expression de la protéine GFP (green fluorescent protein) issue de la méduse Aequorea victoria, et commercialisé aujourd'hui pour l'aquariophilie [12]. Mais la techno- logie des iPS permettra d'utiliser d'autres méthodes de manipulation génétique, en particulier la recombinaison homologue, et de créer des animaux KO différents de la souris. Pour cela, il faudra montrer qu'il est possible d'élargir la liste des animaux dont les cellules sont reprogrammables; cette liste inclut aujourd'hui la souris, le rat, le primate non humain et l'homme [9]. Il sera aussi nécessaire de contourner I'utilisation des rétro- et lentivirus pour la reprogrammation, dont l'intégration dans I'ADN engendre une altération non contrôlée du génome de la cellule cible. Ces obstacles franchis, des iPS issues d'animaux tels que le chien, le mouton ou le porc - pour lesquels des lignées ES indiscutables n'ont pas encore été établies - pourraient faciliter la création de KO de grands animaux, qui seraient de meilleurs modèles précliniques que les rongeurs. $\diamond$

Rat embryonic stem cells. So what?

\section{RÉFÉRENCES}

1. Austin CP, Battey JF, Bradley A, et al. The knockout mouse project. Nat Genet 2004 ; 36 : 921-4.

2. Masson R, Sorg T, Warot X. Génomique fonctionnelle de la souris : la dynamique européenne. Med Sci (Paris) $2007 ; 23: 877-9$.
Figure 1. La dérivation de lignées de cellules عS de rat est maintenant possible grâce à l'utilisation d'une combinaison de trois inhibiteurs chimiques « $3 i$ ». Après manipulation génétique (recombinaison homologue ou autre), l'introduction des cellules pluripotentes ( $\varepsilon S$ ou iPS, induced pluripotent stem cells) dans le blastocyste aboutit à des rats chimères dont certaines cellules, y compris germinales, peuvent être porteuses de la manipulation génétique. Après reproduction des rats chimères entre eux, certains animaux de seconde génération peuvent être homozygotes pour la modification génétique. Grâce aux iPS, il est concevable que cette technologie se décline dans l'avenir à d'autres animaux tels que chien, mouton ou porc, permettant la mise au point de modèles de pathologies humaines à partir de gros animaux. $\mathrm{MCl}$ : masse cellulaire interne.

3. Cohen-Tannoudji M. Prix Nobel de Médecine 2007. Des souris mutantes à façon. Med Sci (Paris) 2007 ; $23: 1159-61$.

4. Ying QL, Wray J, Nichols J, et al. The ground state of embryonic stem cell self-renewal. Nature 2008 ; 453 : 519-23.

5. Coulombel L. Bête comme chou. Med Sci (Paris) 2008 ; $24: 593$.

6. Buehr M, Meek S, Blair K, et al. Capture of authentic embryonic stem cells from rat blastocysts. Cell 2008 $135:$ 1287-98.

7. Li P, Tong C, Mehrian-Shai R, et al. Germline competent embryonic stem cells derived from rat blastocysts. Cell 2008 ; 135 : 1299-310.

8. Coulombel L. Reprogrammation nucléaire d'une cellule différenciée : on efface tout et on recommence. Med Sci (Paris) 2007 ; 23 : 667-70.

9. Trounson A. Rats, cats, and elephants, but still no unicorn: induced pluripotent stem cells from new species. Cell Stem Cell $2009 ; 4: 3-4$.

10. Li W, Wei W, Zhu S, et al. Generation of rat and human induced pluripotent stem cells by combining genetic reprogramming and chemical inhibitors. Cell Stem Cell $2009 ; 4: 16-9$.

11. Liao J, Cui C, Chen S, et al. Generation of induced pluripotent stem cell lines from adult rat cells. Cell Stem Cell $2009 ; 4: 11-5$.

12. Knight J. GloFish casts light on murky policing of transgenic animals. Nature $2003 ; 426: 372$. 